\title{
Minimally Invasive Corrective Osteotomy with the Ilizarov Mini-Fixator for Malunited Fractures of the Phalanges: Technical Note
}

\author{
Yuji Tomori, Mitsuhiko Nanno, Kentaro Sonoki and Tokifumi Majima \\ Department of Orthopedic Surgery, Nippon Medical School Hospital, Tokyo, Japan
}

\begin{abstract}
Posttraumatic malunion of the phalanx with rotational deformity may cause crossing of the finger and impair hand function. Cosmetic disfigurement and severe dysfunction of the fingers require surgical correction, most often via open corrective osteotomies and rigid fixation with plates. However, such an approach requires a longer incision, inevitably results in a scar, and has a higher potential for extensor tendon adhesions, which result in extension lags. In addition, abruption of the periosteum and plating of the phalanges require longer bone healing. This report describes the straightforward, minimally invasive correction of phalangeal malunions with a mini-external fixator. In this procedure, a digital block of the affected finger can be performed. The fully flexed position of all fingers provides accurate correction of phalangeal malunions. Although treatment of phalangeal malunions remains challenging, the present procedure is an alternative solution for phalangeal malunions. (J Nippon Med Sch 2021; 88: 262-266)
\end{abstract}

Key words: finger, malunion, corrective osteotomy, external fixator, Ilizarov mini-fixator

\section{Introduction}

Most fractures of the phalanx are treated non-surgically and usually achieve bone union uneventfully. However, some deformations due to malunion of the fractured phalanx may cause cosmetic deformation or functional impairment. These include crossing of the fingers, disturbance in tendon balance, and reduction of grip strength $^{1-4}$. When cosmetic and functional disability is not acceptable for patients, the deformity must be surgically corrected. A limited number of case series have investigated surgical correction of malunion of the proximal phalan $x^{1-5}$, most often via open corrective osteotomies and rigid fixation with plates. However, such an approach requires a longer incision, inevitably results in a scar, and has a higher potential for extensor tendon adhesions, which result in extension lags. Moreover, abruption of the periosteum and plating of the phalanges require longer bone healing.

In this study, the authors describe a minimally invasive technique for correcting phalangeal malunions with the Ilizarov mini-fixator.

\section{Device}

The Ilizarov mini-fixator (Ito Medical Instruments, Tokyo, Japan)-an external fixator developed by the Russian Ilizarov Scientific Center-was used. This multiplanar fixator can link with all other units three-dimensionally by combining the rod, hinge, and nut; it can also be used for bone elongation ${ }^{6}$. The size of the pin and drill depend on the phalanx where it will be used. The 1.2-mm-diameter and 1.5-mm-diameter dedicated pins are usually used for the middle and proximal phalanges, respectively.

\section{Indications}

External fixation with the Ilizarov mini-fixator is a safe, minimally invasive procedure that provides considerable benefits to almost all patients. This procedure is generally indicated for patients who have malunited fractures of the phalanges, including proximal and middle phalanges, and metacarpals. However, malunions of the metacarpals can be also treated with other procedures, including plates, screws, or wires, without difficulties ${ }^{1-5,7-10}$. Thus, the procedure is generally indicated for malunited fractures of the proximal and middle phalanges.

Correspondence to Yuji Tomori, MD, PhD, Department of Orthopedic Surgery, Nippon Medical School, 1-1-5 Sendagi, Bunkyoku, Tokyo 113-8603, Japan

E-mail: s4064@nms.ac.jp

https://doi.org/10.1272/jnms.JNMS.2021_88-314

Journal Website (https://www.nms.ac.jp/sh/jnms/) 

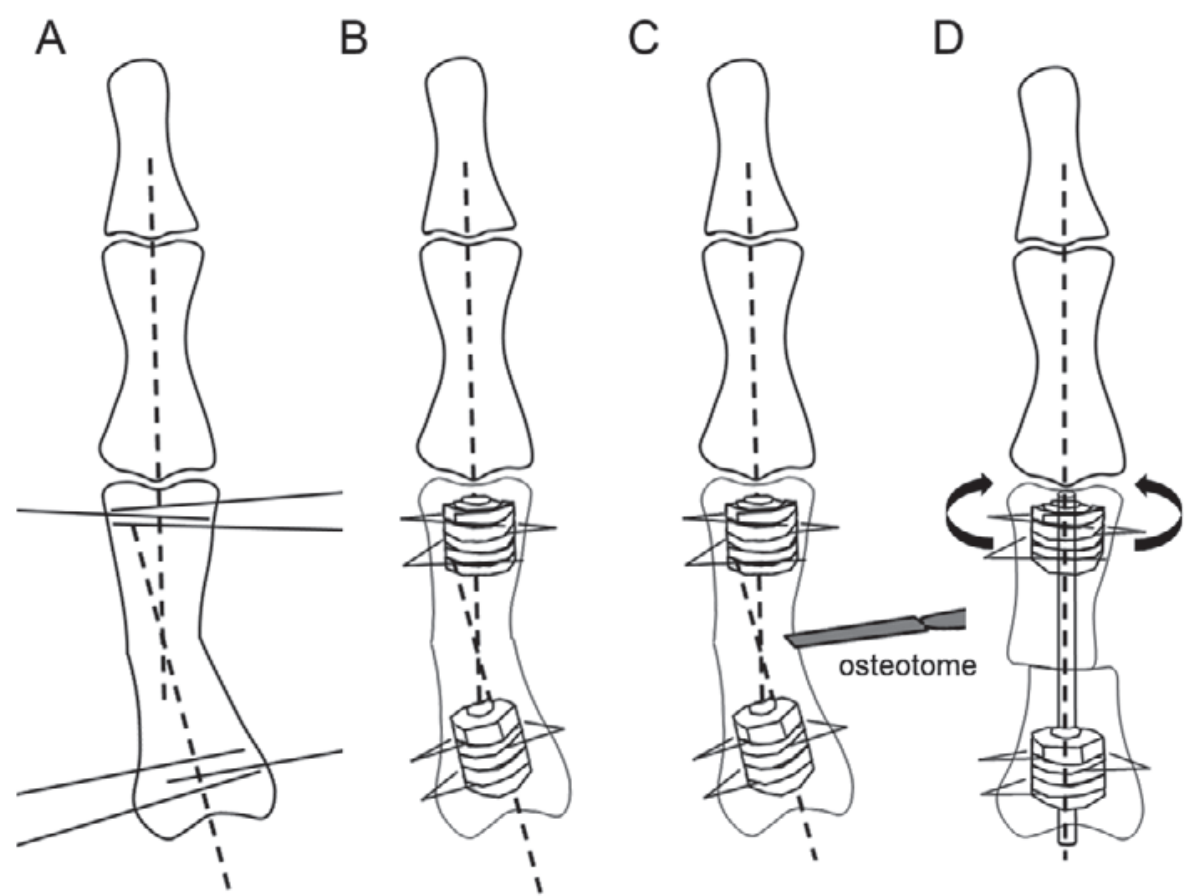

Fig. 1 Minimally invasive corrective osteotomy with the Ilizarov mini-fixator for malunited fractures of the phalanges.

The dedicated pins are inserted into the proximal and distal parts of the presumptive osteotomy site (A). The rod of the fixator is adjusted to make it parallel to the axis of the proximal and distal parts of the proximal phalanx (dotted line). The surgeon then attaches the rod to the Ilizarov mini-fixator units (B). A 10-mm incision is made at the presumptive osteotomy site, and osteotomy is performed through the skin incision with a 5-mm osteotome (C). The mini-fixator is adjusted according to the fully flexed position of the fingers, and each fixator unit is held and fixed in a position in which the alignment of the fingers is corrected (D).

The Ilizarov mini-fixator is contraindicated for some patients. Relative contraindications include noncompliant patients, because they may not return for follow-up or for removal of the device. General contraindications include immunocompromised patients such as those with uncontrolled diabetes mellitus and those who are unable to withstand the procedure.

\section{Surgical Technique}

The patient is placed in a supine position. General anesthesia or digital block is performed in accordance with patient preference. The affected finger is then disinfected, and a pneumonic tourniquet is applied before osteotomy.

The surgeon locates the previous fracture, which is the malunion site, under a fluoroscope and marks the location of both lateral ends of the original fracture line. When the site of the malunion is unclear, the metaphysis of the phalanx is marked. Then, the dedicated pins are inserted into the proximal and distal parts of the presumptive osteotomy site (Fig. 1A). The fixator rod is adjusted to make it parallel to the axis of the proximal and distal parts of the proximal phalanx. The surgeon attaches the rod to the Ilizarov mini-fixator units (Fig. 1B). An approximately $10-\mathrm{mm}$ incision is made at the presumptive site of the osteotomy, and the lateral band is retracted dorsally and protected. The periosteum over the proximal phalange is opened, and transverse osteotomy is performed through the skin incision with a 5-mm osteotome (Fig. 1C). The malunited phalange is resected with lighter tapping with a hummer. Because all deformities of the phalange, including flexion, rotational, and angular deformities, are corrected when all fingers are fully flexed, the mini-fixator is adjusted according to the fully flexed position of the fingers, and each fixator unit is fixed in a position in which the alignment of the finger is corrected (Fig. 1D). When there is instability of the osteotomized site, an additional temporary fixation with Kirschner wires is applied to augment the osteotomized site. A bone graft is not required for this procedure. If the phalangeal bone requires elongation, distraction osteogenesis is performed with the Ilizarov minifixator. 

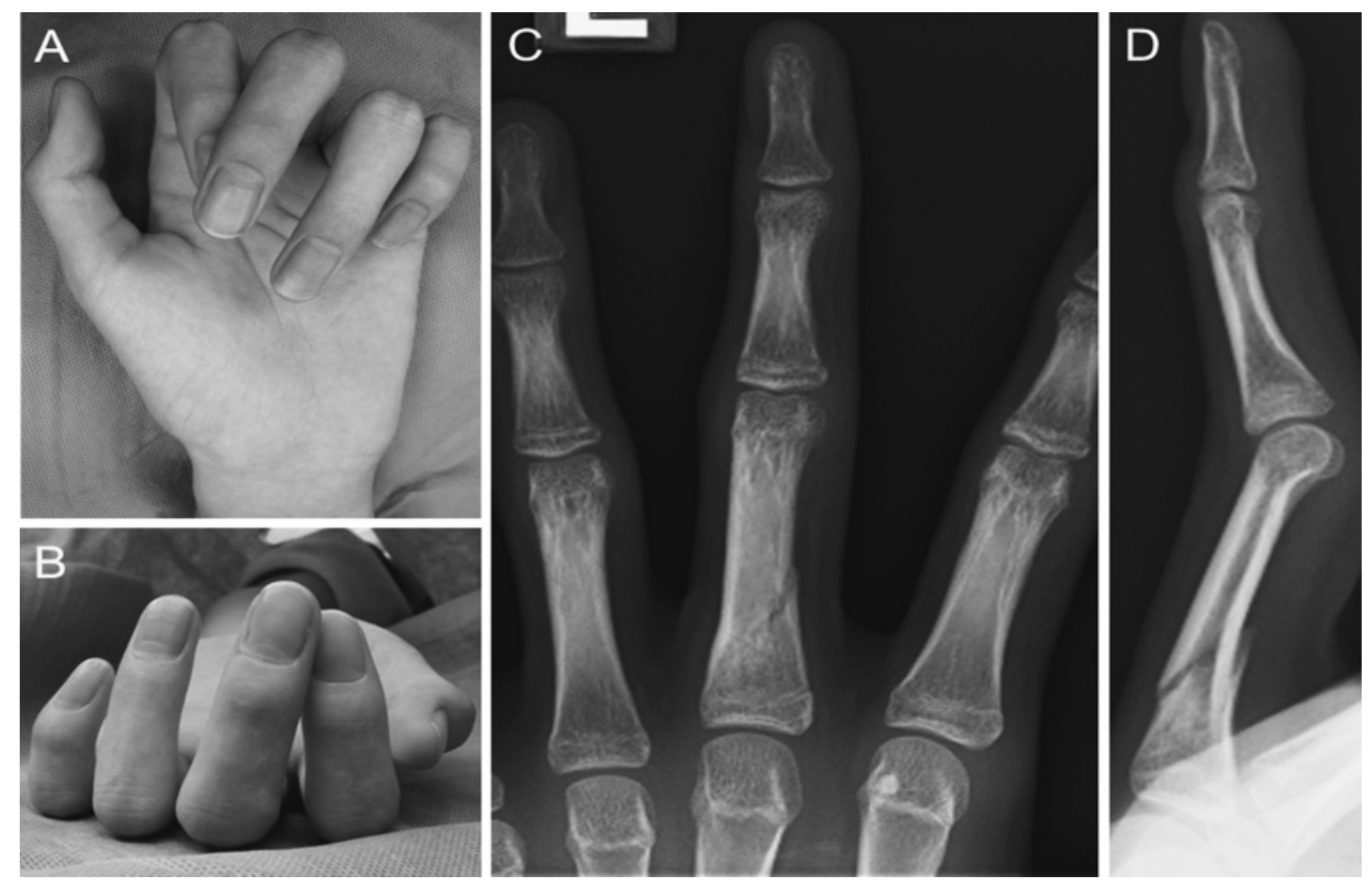

Fig. 2 A photograph of the hand of a 15-year-old right-handed male patient who presented with posttraumatic malunion of the left third proximal phalanx. Physical examination showed ulnar rotation and crossing of the third finger when grasping (A, B). Radiographs showing malunion of the left third proximal phalanx (C: posteroanterior, D: lateral view).

\section{Postoperative Management}

Active range of motion (ROM) exercises are encouraged immediately after surgery. The insertion sites of the dedicated pins are checked once a week. Superficial infection of the insertion sites of the dedicated pins is treated with oral antibiotics. The dedicated pins are removed at an outpatient clinic after 6 weeks, and a knuckle splint is then applied for several weeks if radiographs show inadequate bone callus and fragility of the osteotomized site. Follow-up radiographs are taken every 2 weeks for 8 weeks postoperatively. After that, radiographs are taken monthly until rigid consolidation of the osteotomized site is observed.

\section{Case Presentation}

A 15-year-old right-handed male patient presented with posttraumatic phalangeal malunion. The patient fell from the top of a desk and broke his left third proximal phalanx 3 months previously. He was treated with a volar splint that included the interphalangeal and metacarpophalangeal joints for 4 weeks. During the first visit to the authors' hospital, physical examination showed ulnar rotation and crossing of the third finger when grasping (Fig. 2A, B). Radiographs revealed malunion of the left third proximal phalanx (Fig. 2C, D). Minimally invasive corrective osteotomy with the use of the Ilizarov minifixator was performed (Fig. 3A, B). After 6 weeks, the fixator was removed. Follow-up radiographs taken 6 weeks after correction surgery showed union of the osteotomy, but the fragility of the bone union was suspected (Fig. 3C, D). A knuckle splint was applied for 4 weeks after removal of the fixator. The patient progressed in his activities during the next 6 weeks and resumed full activity, without pain, at 3 months postoperatively. At the latest follow-up, 24 months after surgery, radiographs showed a well-corrected rotational deformity (Fig. 3E, F). The patient has no functional deficits and barely discernible stab incision scars. In addition, full flexion and extension of the finger were obtained (Fig. 3 G, H, I).

\section{Discussion}

Most malunions of the phalanx are a combination of angular, rotational, and shortening deformities ${ }^{1-5}$. Thus, surgical correction of phalangeal malunions is challenging and technically demanding ${ }^{1-5}$. Moreover, few studies have examined corrective osteotomy to fix phalangeal malunions, and most had small samples and short follow-up periods $^{1-5,7-10}$.

A closing-wedge osteotomy or opening-wedge osteot- 

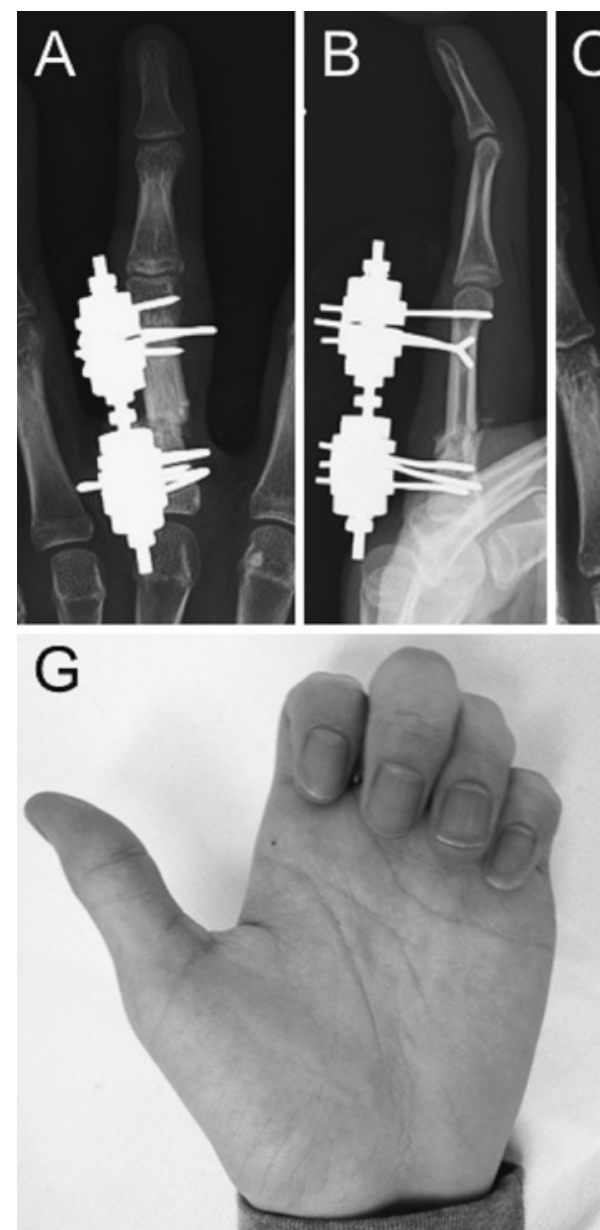
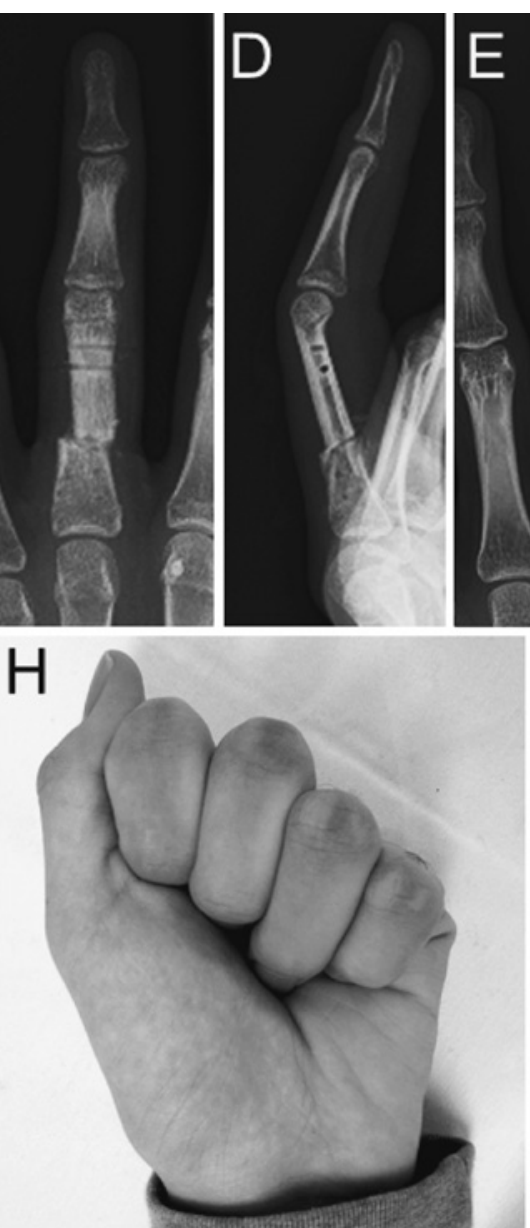
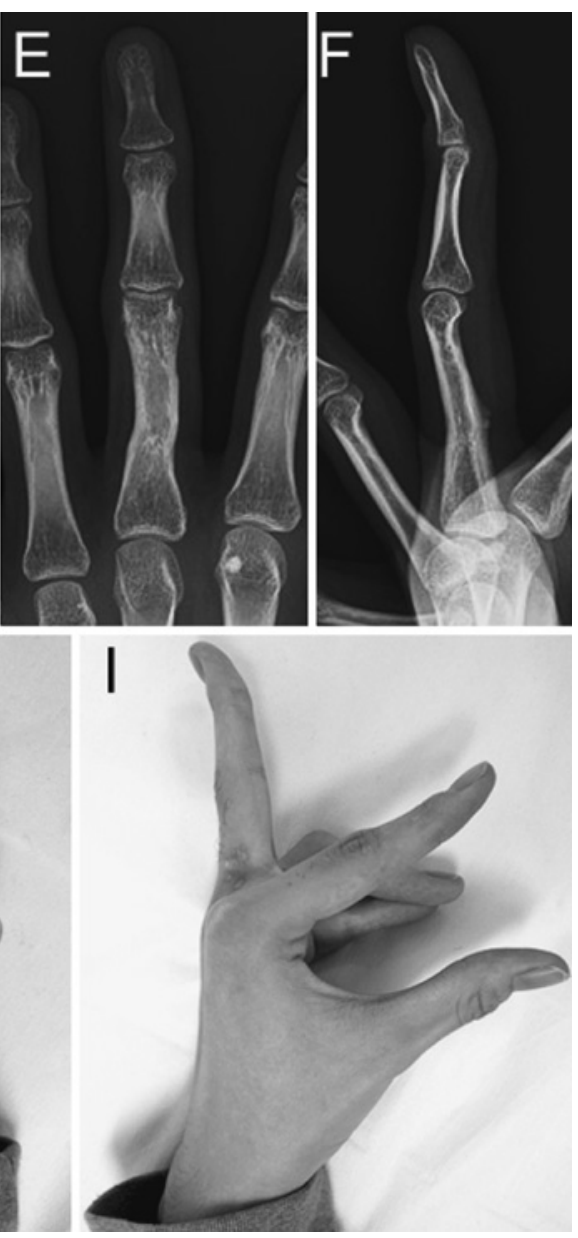

Fig. 3 Postoperative radiographs showing the osteotomy line and oblique insertion of the dedicated pins (A: posteroanterior, B: lateral view). Follow-up radiographs taken 6 weeks after correction surgery showed union of the osteotomy. However, fragility of the bone union was suspected, and a knuckle splint was applied for 4 weeks after removal of the fixator (C: posteroanterior, D: lateral view). At the latest follow-up, 24 months after surgery, radiographs show a well-corrected rotational deformity (E: posteroanterior, F: lateral view). Photographs of the right hand with fingers in flexion $(\mathrm{G}, \mathrm{H})$ and extension $(\mathrm{I})$.

omy at the fracture site has been utilized for correction ${ }^{5}$. A previous cohort study reported that incomplete opening-wedge osteotomies for posttraumatic malunions was the optimal method to correct lateral angulation, flexion, and extension deformities ${ }^{2}$. For anatomical correction, a corrective osteotomy should be performed at the fracture site. However, an osteotomy on the site of the original fracture followed by internal fixation could result in limitation of digital ROM because it causes additional adhesions between the flexor and extensor tendons that surround the proximal phalanx. In addition, adhesion development in the flexor and extensor systems and shortening of the phalanges could result in extension $\operatorname{lags}{ }^{7,8}$.

Some authors have reported corrections at the base of the metacarpal of the affected finger ${ }^{4,10}$. A metacarpal osteotomy of the affected finger may provide rotational correction without potential adhesions but may not pro- vide precise correction when angulation deformities accompany malrotation'. In addition, an anatomical limitation of metacarpal rotational correction was reported in a cadaveric study ${ }^{10}$.

As regards internal fixation of the osteotomized site, most authors have reported corrective osteotomy followed by internal fixation with plates or screws $s^{1-5,7-10}$. However, even osteosynthesis of fresh phalangeal fractures can occasionally cause adhesions between the flexor and extensor tendons, resulting in ROM restrictions and flexion/extension lags ${ }^{7,8}$. Moreover, internal fixation of the phalanx with plates or screws can cause residual pain or skin irritation, requiring removal surgery.

In the present method, a digital block of the affected finger can be performed, and full flexion of all fingers provides precise correction of the malunion of the phalanx. Furthermore, this method avoids dissection of surrounding tendons, and thus additional adhesions. More- 
over, the Ilizarov mini-fixator is feasible for performing distraction osteogenesis when there is shortening of the phalan $x^{6}$. Nevertheless, this method has several drawbacks, including the risk of pin infection and interference in activities of daily living.

Although further study is needed, this minimally invasive correction procedure using the Ilizarov mini-fixator is simple and reproducible and is an alternative solution for phalangeal malunions.

Conflict of Interest: The authors declare no conflict of interest regarding the publication of this article.

\section{References}

1. Potenza V, De Luna V, Maglione P, Garro L, Farsetti P, Caterini R. Post-traumatic malunion of the proximal phalanx of the finger. Medium-term results in 24 cases treated by "in situ" osteotomy. Open Orthop J. 2012;6: 468-72.

2. Büchler U, Gupta A, Ruf S. Corrective osteotomy for post-traumatic malunion of the phalanges in the hand. J Hand Surg Br. 1996;21:33-42.

3. Ring D. Malunion and nonunion of the metacarpals and phalanges. Instr Course Lect. 2006;55:121-8.

4. Weckesser E. Rotational osteotomy of the metacarpal for overlapping fingers. J Bone Joint Surg Am. 1965;47:751-6.

5. van der Lei B, de Jonge J, Robinson PH, Klasen HJ. Correction osteotomies of phalanges and metacarpals for ro- tational and angular malunion: a long-term follow-up and a review of the literature. J Trauma. 1993;35:902-8.

6. Sawaizumi $T$, Ito $H$. Lengthening of the amputation stumps of the distal phalanges using the modified Ilizarov method. J Hand Surg Am. 2003;28:316-22.

7. Vahey JW, Wegner DA, Hastings H. Effect of proximal phalangeal fracture deformity on extensor tendon function. J Hand Surg Am. 1998;23:673-81.

8. Yong FC, Tan SH, Tow BP, Teoh LC. Trapezoid rotational bone graft osteotomy for metacarpal and phalangeal fracture malunion. J Hand Surg Eur Vol. 2007;32:282-8.

9. Trumble T, Gilbert M. In situ osteotomy for extra-articular malunion of the proximal phalanx. J Hand Surg Am. 1998;23:821-6.

10. Gross MS, Gelberman RH. Metacarpal rotational osteotomy. J Hand Surg Am. 1985;10:105-8.

(Received, April 29, 2020)

(Accepted, July 31, 2020)

(J-STAGE Advance Publication, August 31, 2020)

Journal of Nippon Medical School has adopted the Creative Commons Attribution-NonCommercial-NoDerivatives 4.0 International License (https://creativecommons.org/licenses/by-nc-nd/4.0/) for this article. The Medical Association of Nippon Medical School remains the copyright holder of all articles. Anyone may download, reuse, copy, reprint, or distribute articles for non-profit purposes under this license, on condition that the authors of the articles are properly credited. 